Comment. Math. Helv. 78 (2003) 447-462

0010-2571/03/030447-16

DOI $10.1007 / \mathrm{s} 00014-003-0762-0$ (c) 2003 Birkhäuser Verlag, Basel

Commentarii Mathematici Helvetici

\title{
Elementary modifications and line configurations in $\mathbb{P}^{2}$
}

\author{
Henry K. Schenck
}

\begin{abstract}
Associated to a projective arrangement of hyperplanes $\mathcal{A} \subseteq \mathbb{P}^{n}$ is the module $D(\mathcal{A})$, which consists of derivations tangent to $\mathcal{A}$. We study $D(\mathcal{A})$ when $\mathcal{A}$ is a configuration of lines in $\mathbb{P}^{2}$. In this setting, we relate the deletion/restriction construction used in the study of hyperplane arrangements to elementary modifications of bundles. This allows us to obtain bounds on the Castelnuovo-Mumford regularity of $D(\mathcal{A})$. We also give simple combinatorial conditions for the associated bundle to be stable, and describe its jump lines. These regularity bounds and stability considerations impose constraints on Terao's conjecture.
\end{abstract}

Mathematics Subject Classification (2000). 52B30, 14J60, 14Q10.

Keywords. Hyperplane arrangement, vector bundle, Castelnuovo-Mumford regularity, stability, jump locus.

\section{Introduction}

In this paper we investigate the connection between a standard construction in algebraic geometry (elementary modifications of bundles) and a standard construction in the study of hyperplane arrangements (the deletion/restriction operation). In the setting of line configurations in $\mathbb{P}^{2}$, it turns out that they are the same thing. Given a two bundle $V$ and line $L$, if $V$ sits in a modification

$$
0 \longrightarrow W \longrightarrow V \longrightarrow i_{*} \mathcal{O}_{L}(a) \longrightarrow 0,
$$

then understanding $V$ means understanding $W$ and $a$ (here $i$ is the inclusion of $L$ in $\left.\mathbb{P}^{2}\right)$. For line configurations, the twist $a$ has a simple combinatorial meaning, and the long exact sequence in cohomology yields information about the arrangement. In particular, it gives a bound on the Castelnuovo-Mumford regularity of the module of derivations tangent to $\mathcal{A}$.

In $\S 2$ we give a quick review of the fundamental objects: syzygy modules and hyperplane arrangements. The module of $\mathcal{A}$ derivations is denoted $D(\mathcal{A})$, it consists of derivations of $\mathbb{P}^{n}$ tangent to $\mathcal{A}$. The syzygy module on the Jacobian ideal

\footnotetext{
This work was completed while the author was an NSF postdoctoral fellow at Harvard University.
} 
of the singular hypersurface $\mathcal{A}$ is a summand of $D(\mathcal{A})$, hence the connection. In $\S 3$ we discuss elementary modifications and Castelnuovo-Mumford regularity, and in $\S 4$ turn our attention to stability. We close with an application of these results to Terao's conjecture that the freeness of $D(\mathcal{A})$ depends only on the combinatorics of the arrangement.

\section{Zero dimensional subschemes and line configurations}

Let $R=k\left[x_{0}, x_{1}, x_{2}\right]$ and $I=\left\langle s_{1}, \ldots, s_{k}\right\rangle \subseteq R_{m}$ a codimension two ideal; $I$ defines a map:

$$
\mathcal{O}_{\mathbb{P}^{2}}^{k} \stackrel{I}{\longrightarrow} \mathcal{O}_{\mathbb{P}^{2}}(m) \text {. }
$$

Let $\mathcal{D}$ denote the sheaf associated to the module of syzygies on $I$. $\mathcal{D} \simeq \oplus \mathcal{O}\left(\gamma_{i}\right)$ iff $I$ is saturated. Since $\mathcal{D}$ is a second syzygy sheaf, $\mathcal{D}$ is locally free. Let $Z$ be the scheme defined by $I$; the following lemma follows from standard properties of Chern classes:

Lemma 2.1. The Chern classes of $\mathcal{D}$ are $c_{1}(\mathcal{D})=-m, c_{2}(\mathcal{D})=m^{2}-\operatorname{deg}(Z)$.

In [18], Serre describes a method of constructing a rank two vector bundle $\mathcal{F}$ on $\mathbb{P}^{n}$ from a codimension two local complete intersection $Y$ with ideal sheaf $\mathcal{I}_{Y}$. If the determinant bundle of the normal bundle of $Y$ extends to a bundle on $\mathbb{P}^{n}$ :

$$
\left.\operatorname{det} \mathcal{N}_{Y / \mathbb{P}^{n}} \simeq \mathcal{O}_{\mathbb{P}^{n}}(m)\right|_{Y}
$$

then there is a rank two bundle $\mathcal{F}$ on $\mathbb{P}^{n}$ with section $s$, which induces the short exact sequence:

$$
0 \longrightarrow \mathcal{O}_{\mathbb{P} n} \stackrel{\cdot s}{\longrightarrow} \mathcal{F} \longrightarrow \mathcal{I}_{Y}(m) \longrightarrow 0 .
$$

The Chern classes of $\mathcal{F}$ are given by $c_{1}(\mathcal{F})=m$ and $c_{2}(\mathcal{F})=\operatorname{deg} Y$. If $Z$ is a local complete intersection and the bundle $\mathcal{F}$ exists, then the bundles $\mathcal{D}$ and $\mathcal{F}$ are related by the exact sequence:

$$
0 \longrightarrow \mathcal{D} \longrightarrow \mathcal{O}^{k+1} \longrightarrow \mathcal{F} \longrightarrow 0 \text {. }
$$

Let char $k=0$ and let $Q \in R_{m+1}$ be a reduced polynomial; the role of $I$ will be played by the Jacobian ideal of $Q$. For the remainder of the paper we restrict our attention to the case where $Q$ is a product of distinct linear forms, although many of the results can be generalized. We begin with some facts about hyperplane arrangements; for more information see Orlik and Terao [14].

A hyperplane arrangement $\mathcal{A}$ is a finite collection of codimension one linear subspaces of a fixed vector space $\mathrm{V} . \mathcal{A}$ is central if each hyperplane contains the origin $\mathbf{0}$ of $\mathrm{V}$. The intersection lattice $L_{\mathcal{A}}$ of $\mathcal{A}$ consists of the intersections of the elements of $\mathcal{A}$; the rank of $x \in L_{\mathcal{A}}$ is simply the codimension of $x . \mathrm{V}$ is the lattice element $\hat{0}$; the rank one elements are the hyperplanes themselves. $\mathcal{A}$ is called 
essential if rank $L_{\mathcal{A}}=\operatorname{dim} V$. Henceforth, $\mathcal{A}$ will be an essential, central three arrangement with $|\mathcal{A}|=d$; i.e. a set of $d$ lines in $\mathbb{P}_{k}^{2}$.

Definition 2.2. The Möbius function $\mu: L_{\mathcal{A}} \longrightarrow \mathbb{Z}$ is defined by

$$
\begin{aligned}
& \mu(\hat{0})= \\
& \mu(t)=-\sum_{s<t} \mu(s), \text { if } \hat{0}<t
\end{aligned}
$$

Definition 2.3. The Poincaré polynomial $\pi(\mathcal{A}, t)=\sum_{x \in L_{\mathcal{A}}} \mu(x) \cdot(-t)^{\operatorname{rank}(x)}$.

It follows directly from the definitions above that for such an arrangement,

$$
\pi(\mathcal{A}, t)=(1+t)\left(1+(d-1) t+\left(\sum_{\substack{x \in L_{\mathcal{A}} \\ \operatorname{rank}(x)=2}} \mu(x)-d+1\right) t^{2}\right) .
$$

Let $Q$ be a reduced polynomial defining $\mathcal{A}$ and $J_{Q}$ the Jacobian ideal of $Q$. The next lemma gives an easy proof of the main result of [16]:

Lemma 2.4. The Jacobian ideal of a line arrangement in $\mathbb{P}^{2}$ is a local complete intersection.

Proof. Localization, the product rule, and Euler's relation.

Theorem 2.5. If $\mathcal{A}$ is a line arrangement defined by $Q$ and $\mathcal{D}$ is the syzygy bundle of $J_{Q}$, then

$$
\pi(\mathcal{A}, t)=(1+t) \cdot c_{t}\left(\mathcal{D}^{\vee}\right)
$$

where $c_{t}$ is the Chern polynomial.

Proof. Lemma 2.1 implies that

$$
c_{t}\left(\mathcal{D}^{\vee}\right)=1+(d-1) t+\left((d-1)^{2}-\operatorname{deg} J_{Q}\right) t^{2},
$$

and by Lemma 2.4 we have

$$
\operatorname{deg} J_{Q}=\sum_{\substack{x \in L_{\mathcal{A}} \\ \operatorname{rank}(x)=2}} \mu(x)^{2} .
$$

Now use the identity:

$$
\left(\begin{array}{l}
d \\
2
\end{array}\right)=\sum_{\substack{x \in L_{\mathcal{A}} \\
\operatorname{rank}(x)=2}}\left(\begin{array}{c}
\mu(x)+1 \\
2
\end{array}\right) .
$$

The motivation for the previous theorem is Terao's celebrated freeness theorem [20]. Let $\mathcal{A} \subseteq \mathbb{P}^{n}$ be an arrangement with defining polynomial $Q \in R=$ $k\left[x_{0}, \ldots, x_{n}\right]$; the module of derivations tangent to $\mathcal{A}$ is defined as: 
Definition 2.6.

$$
D(\mathcal{A})=\left\{\theta \in \operatorname{Der}_{k}(R) \mid \theta(Q) \in\langle Q\rangle\right\}
$$

Terao's theorem is that if $D(\mathcal{A})$ is free, then the Poincaré polynomial factors as $\Pi_{i=0}^{n}\left(1+a_{i} t\right)$, where $a_{i}$ are the degrees of a set of homogeneous generators of $D(\mathcal{A})$. If char $k=0$ then $D(\mathcal{A}) \simeq D_{0} \oplus R(-1)$, where $R(-1)$ is generated by the Euler derivation and $D_{0}$ is the module of syzygies on $J_{Q}$. Henceforth, $\mathcal{D}$ will be the sheaf associated to $D_{0}$.

\section{Elementary modifications and Castelnuovo-Mumford regular- ity}

A triple of arrangements $\left(\mathcal{A}^{\prime}, \mathcal{A}, \mathcal{A}^{\prime \prime}\right)$ consists of an arrangement $\mathcal{A}$ and choice of distinguished hyperplane $H \in \mathcal{A}$ such that $\mathcal{A}^{\prime}=\mathcal{A}-H$ and $\mathcal{A}^{\prime \prime}=\left.\mathcal{A}\right|_{H} \cdot \mathcal{A}^{\prime}$ is called the deletion of $\mathcal{A}$ and $\mathcal{A}^{\prime \prime}$ the restriction of $\mathcal{A}$ with respect to $H$. Of course, the invariants of the elements of a triple are closely related, and deletion-restriction is often a valuable tool for inductive proofs. For the module of derivations, we have:

Proposition 3.1. (Proposition 4.45 of [14].) There is an exact sequence:

$$
0 \longrightarrow D\left(\mathcal{A}^{\prime}\right)(-1) \stackrel{\cdot H}{\longrightarrow} D(\mathcal{A}) \longrightarrow D\left(\mathcal{A}^{\prime \prime}\right)
$$

Orlik and Terao give an example of a line arrangement for which the above sequence is not right exact (see example 4.56 of [14] - this is example I in the next section, with the role of $H$ played by $\{z=0\})$. Recall the definition of an elementary modification (see [8]): Let $X$ be a smooth variety, $Y$ an effective divisor on $X, Y \stackrel{i}{\hookrightarrow} X$. Let $V$ be a rank two bundle on $X, L$ a line bundle on $Y$, and suppose $V \rightarrow i_{*} L \rightarrow 0$. Then the kernel $W$ of the map is also a rank two bundle on $X$, with $c_{1}(W)=c_{1}(V)-Y$ and $c_{2}(W)=c_{2}(V)-c_{1}(V) \cdot Y+i_{*} c_{1}(L)$.

Theorem 3.2. Let $\mathcal{A}$ be an arrangement of lines in $\mathbb{P}^{2}$. If $\left(\mathcal{A}^{\prime}, \mathcal{A}, \mathcal{A}^{\prime \prime}\right)$ is a triple, and $i: H \simeq \mathbb{P}^{1} \hookrightarrow \mathbb{P}^{2}$, then the sequence of sheaves corresponding to Proposition 3.1 (with Euler derivations pruned off) is an elementary modification, i.e.

$$
0 \longrightarrow \mathcal{D}^{\prime}(-1) \longrightarrow \mathcal{D} \longrightarrow i_{*} \mathcal{D}^{\prime \prime} \longrightarrow 0
$$

is exact.

Proof. This follows since

$$
i_{*} \mathcal{D}^{\prime \prime} \simeq \mathcal{O}_{H}\left(1-\left|\mathcal{A}^{\prime \prime}\right|\right) .
$$

Now use the Hirzebruch-Riemann-Roch theorem and Serre vanishing to convert the Chern polynomials to Hilbert polynomials, and compute. 
An important measure of the complexity of a coherent sheaf on $\mathbb{P}^{n}$ is the Castelnuovo-Mumford regularity:

Definition 3.3. A coherent sheaf $\mathcal{F}$ on $\mathbb{P}^{n}$ is $m$-regular $(\operatorname{reg}(\mathcal{F})=m)$ if

$$
H^{i}(\mathcal{F}(m-i))=0 \forall i \geq 1 .
$$

The exact sequence $0 \longrightarrow \mathcal{D}^{\prime}(-1) \longrightarrow \mathcal{D} \longrightarrow i_{*} \mathcal{D}^{\prime \prime} \longrightarrow 0$ gives us a good way to bound the Castelnuovo-Mumford regularity of line arrangements.

Theorem 3.4. For a triple $\left(\mathcal{A}^{\prime}, \mathcal{A}, \mathcal{A}^{\prime \prime}\right)$ of line arrangements,

$$
\operatorname{reg}(\mathcal{D}) \leq \max \left\{\operatorname{reg}\left(\mathcal{D}^{\prime}\right)+1,\left|\mathcal{A}^{\prime \prime}\right|-1\right\} .
$$

Proof. Follows from the long exact sequence in cohomology.

Corollary 3.5. For an arrangement on $d$ lines, $\operatorname{reg}(\mathcal{D}) \leq d-2$. This is tight for generic arrangements.

Since the sheaf $\mathcal{D}$ is reflexive, it corresponds to a bundle on $\mathbb{P}^{2}$ and ([12])

$$
D_{0} \simeq \bigoplus_{i} H^{0}(\mathcal{D}(i))
$$

For an arrangement of $d$ lines, this means that the minimal free resolution of $D_{0}$ is:

$$
0 \longrightarrow \bigoplus_{j=1}^{m-2} R\left(-\beta_{j}\right) \longrightarrow \bigoplus_{i=1}^{m} R\left(-\alpha_{i}\right) \longrightarrow D_{0} \longrightarrow 0
$$

where the $\alpha_{i}$ are at most $d-2$ and the $\beta_{j}$ are at most $d-1$. In [26], Ziegler obtains results on the generators for $D_{0}^{\vee}$. For hypersurfaces with only isolated singularities, Choudary and Dimca [3] give a bound; for a (reduced, singular) degree $d$ curve in $\mathbb{P}^{2}$, the regularity of $D_{0}$ is at most $2 d-4$. Thus, for line arrangements, the bound above is better than existing results.

The entire free resolution of $D_{0}$ for a generic arrangement is given in [15] and [22]. In [24], Yuzvinsky gives a set of generators for a submodule of $D_{0}^{\vee}$; these generators are determined by $L_{2}(\mathcal{A})$. When the third relation space vanishes, they actually generate the entire module (this generalizes the results of Ziegler mentioned earlier). Even in the case of line configurations, there are examples (e.g. the Braid arrangement) where this space does not vanish. However, the maximal number of generators of $D_{0}$ is bounded by $d-1$ (which is attained by generic arrangements). For one proof of this, see Jiang and Feng [11], §4.2. Finally, we note that Derksen and Sidman [4] have recently obtained regularity bounds on $D(\mathcal{A})$ for higher dimensional arrangements. 


\section{Stability and jump loci}

In this section, we consider the stability and jump loci of the bundle $\mathcal{D}$ obtained from an arrangement of $d$ lines; the point is that we can often construct stable bundles with prescribed jumping lines. For generic arrangements these questions were studied by Dolgachev and Kapranov in [5], [6]. We want to investigate $\mathcal{D}$ when the arrangement is nongeneric; the tool will be the short exact sequence of the last section. We first recall a few standard results about vector bundles on $\mathbb{P}^{n}$, referring for proofs to the book of Okonek, Schneider and Spindler [13].

Definition 4.1. Let $\mathcal{M}$ be a bundle on $\mathbb{P}^{n}$. The slope of $\mathcal{M}$ is defined as

$$
\operatorname{slope}(M)=\frac{c_{1}(\mathcal{M})}{\operatorname{rk} \mathcal{M}} .
$$

A key concept in the study of bundles on $\mathbb{P}^{n}$ is stability:

Definition 4.2. A bundle $\mathcal{M}$ on $\mathbb{P}^{n}$ is stable if for all subsheaves $\mathcal{N} \subseteq \mathcal{M}$ with $0<\operatorname{rk} \mathcal{N}<\operatorname{rk} \mathcal{M}$

$$
\operatorname{slope}(\mathcal{N})<\operatorname{slope}(\mathcal{M})
$$

and semistable if

$$
\operatorname{slope}(\mathcal{N}) \leq \operatorname{slope}(\mathcal{M})
$$

If $\mathcal{M}$ is a stable two bundle on $\mathbb{P}^{2}$, then Schwarzenberger [17] showed that $c_{1}(\mathcal{M})^{2}<4 c_{2}(\mathcal{M})$ (this was generalized by Bogomolov [2]). Thus, a necessary condition for stability of $\mathcal{D}$ is that degree $J_{Q}<\frac{3}{4}(d-1)^{2}$. The degree of the Jacobian ideal ranges from $\left(\begin{array}{l}d \\ 2\end{array}\right)$ (achieved for generic line arrangements) to $d^{2}-$ $3 d+3$ (achieved for arrangements with $d-1$ lines through a point, and one other line in general position, this class of arrangements has $\mathcal{D} \simeq \mathcal{O}(-1) \oplus \mathcal{O}(-d+2)$ ). Basically, as the degree of the Jacobian ideal gets large, $\mathcal{D}$ has less chance of being stable.

For generic arrangements $\mathcal{A}$ in $\mathbb{P}^{n}$, Dolgachev and Kapranov prove that the bundle of meromorphic one forms with logarithmic pole along $\mathcal{A}$ is stable (in the setting of generic line arrangements this bundle is dual to a twist of $\mathcal{D}$, see [12]), they also prove that the map which associates to a set of $d$ generic hyperplanes the corresponding bundle is generically injective if $d \geq 2 n+3$. Since $\operatorname{dim} \mathcal{M}_{\mathbb{P}^{2}}\left(c_{1}, c_{2}\right)=$ $4 c_{2}-c_{1}^{2}-3$ this map has no chance of having Zariski dense image if the number of lines is large (if $d=6$ Dolgachev and Kapranov show that it is). First, a few definitions. The normalization $\mathcal{F}_{\text {norm }}$ of a rank two bundle $\mathcal{F}$ is $\mathcal{F}(i)$, where $i$ is chosen so that $c_{1}(\mathcal{F}(i)) \in\{0,-1\}$, i.e.

Definition 4.3. Let $\mathcal{F}$ be a rank two bundle on $\mathbb{P}^{2}$. Then $\mathcal{F}_{\text {norm }}=\mathcal{F}\left(k_{\mathcal{F}}\right)$, where $k_{\mathcal{F}}=\frac{-c_{1}(\mathcal{F})}{2}$ if $c_{1}(\mathcal{F})$ is even, and $-\frac{c_{1}(\mathcal{F})+1}{2}$ if $c_{1}(\mathcal{F})$ is odd. 
Lemma 4.4. A reflexive sheaf $\mathcal{F}$ of rank two over $\mathbb{P}^{n}$ is stable iff $H^{0}\left(\mathcal{F}_{\text {norm }}\right)=0$. If $c_{1}(\mathcal{F})$ is even, then $\mathcal{F}$ is semistable iff $H^{0}\left(\mathcal{F}_{\text {norm }}(-1)\right)=0$. If $c_{1}(\mathcal{F})$ is odd, semistable and stable coincide.

Theorem 4.5. Let $\mathcal{A}$ be an arrangement of $d$ lines in $\mathrm{P}^{2}, H$ any line in $\mathcal{A}$, and $\left(\mathcal{A}^{\prime}, \mathcal{A}, \mathcal{A}^{\prime \prime}\right)$ the corresponding triple (notice that the restriction to $H$ ignores multiplicities, so $\left.\left|\mathcal{A}^{\prime \prime}\right|=\left|\mathcal{A}^{\prime} \cap H\right|\right)$. Then

If $d$ is odd, then $\mathcal{D}$ is stable if $\mathcal{D}^{\prime}$ is stable and $\left|\mathcal{A}^{\prime \prime}\right|>\frac{d+1}{2}$.

If $d$ is odd, then $\mathcal{D}$ is semistable if $\mathcal{D}^{\prime}$ is semistable and $\left|\mathcal{A}^{\prime \prime}\right|>\frac{d-1}{2}$.

If $d$ is even, then $\mathcal{D}$ is stable if $\mathcal{D}^{\prime}$ is semistable and $\left|\mathcal{A}^{\prime \prime}\right|>\frac{d}{2}$.

Proof. The proofs are similar so we prove the first statement. Since $d$ is odd, $k_{\mathcal{D}}=\frac{d-1}{2}$ and $k_{\mathcal{D}^{\prime}}=k_{\mathcal{D}}-1$. By Lemma $4.4 \mathcal{D}$ is stable iff $H^{0}\left(\mathcal{D}_{\text {norm }}\right)=0$ so it is necessary that $H^{0}\left(\mathcal{D}^{\prime}(-1) \otimes \mathcal{O}\left(k_{\mathcal{D}}\right)\right)=H^{0}\left(\mathcal{D}_{\text {norm }}^{\prime}\right)=0$. The simplest sufficiency criterion is then that $H^{0}\left(i_{*} \mathcal{D}^{\prime \prime} \otimes \mathcal{O}\left(k_{\mathcal{D}}\right)\right)=0$; from the exact sequence

$$
0 \longrightarrow \mathcal{O}\left(-\left|\mathcal{A}^{\prime \prime}\right|\right) \longrightarrow \mathcal{O}\left(1-\left|\mathcal{A}^{\prime \prime}\right|\right) \longrightarrow i_{*} \mathcal{D}^{\prime \prime} \longrightarrow 0
$$

we have that $H^{0}\left(i_{*} \mathcal{D}^{\prime \prime} \otimes \mathcal{O}\left(k_{\mathcal{D}}\right)\right)=0$ iff $\left|\mathcal{A}^{\prime \prime}\right|>\frac{d+1}{2}$.

An iff criterion for stability can be formulated in the obvious fashion: $\mathcal{D}$ is stable iff $H^{0}\left(\mathcal{D}^{\prime}(-1) \otimes \mathcal{O}\left(k_{\mathcal{D}}\right)\right)=0$ and the connecting map $H^{0}\left(i_{*} \mathcal{D}^{\prime \prime} \otimes \mathcal{O}\left(k_{\mathcal{D}}\right)\right) \longrightarrow$ $H^{1}\left(\mathcal{D}^{\prime}(-1) \otimes \mathcal{O}\left(k_{\mathcal{D}}\right)\right)$ is an inclusion. This can be computed in any particular instance, but is not a criterion which is easy to apply; the point of Theorem 4.5 is that if one adds a line $H$ to a configuration $\mathcal{A}^{\prime}$ for which $\mathcal{D}^{\prime}$ is stable, then as long as no more than (roughly) half of the lines become redundant on restricting to $H$, the new configuration is also stable.

Next, we study the jump loci of these bundles. By a theorem of Grothendieck, any bundle on $\mathbb{P}^{1}$ splits as a direct sum of line bundles; in particular, for a rank two bundle $V$ on $\mathbb{P}^{2}$ and line $L$

$$
\left.V\right|_{L} \simeq \mathcal{O}_{L}\left(a_{1}\right) \oplus \mathcal{O}_{L}\left(a_{2}\right)
$$

In the Grassmannian of lines on $\mathbb{P}^{2} \simeq \mathbb{P}^{2^{\vee}}$, there is a nonempty, Zariski open subset where $\left(a_{1}, a_{2}\right)$ is constant; the complement of this subset is the jump locus $j_{V}$ of $V$. By semicontinuity and the Grauert-Mülich theorem, we have

Definition 4.6. For a normalized, semistable two bundle $V$ on $\mathbb{P}^{2}$

$$
j_{V}=\left\{L \in \mathbb{P}^{2^{\vee}} \mid H^{0}\left(\left.V(-1)\right|_{L}\right) \neq 0\right\} .
$$

We now describe the jump lines of $\mathcal{D}$. The method works in general if we have a modification $V \longrightarrow i_{*} \mathcal{O}_{L}(a) \longrightarrow 0$. In, [1], Barth proved that if $V$ is a semistable two bundle on $\mathbb{P}^{n}$ with $c_{1}(V)=0$, then $j_{V}$ is purely one codimensional, and is supported on a divisor of degree $c_{2}(V)$. If $c_{1}(V)=-1$, then in general $V$ has only 
a finite number of jump lines; in [10] Hulek introduced the notion of a jump line of the second kind:

$$
j_{V}^{2}=\left\{L \in \mathbb{P}^{2^{\vee}} \mid H^{0}\left(\left.V\right|_{L^{2}}\right) \neq 0\right\}
$$

and proved that if $V$ is a semistable two bundle on $\mathbb{P}^{n}$ with $c_{1}(V)=-1$, then $j_{V}^{2}$ is a curve of degree $2\left(c_{2}(V)-1\right)$, and $j_{V} \subseteq \operatorname{Sing}\left(j_{V}^{2}\right)$. We begin by asking which of the lines of the arrangement are contained in $j_{\mathcal{D}}$. Let $\left(\mathcal{A}^{\prime}, \mathcal{A}, \mathcal{A}^{\prime \prime}\right)$ be a triple with respect to a line $H$, put $L=H$.

Theorem 4.7. Let $\mathcal{A}$ be an arrangement of d lines, $L \in \mathcal{A}$ and $\mathcal{A}^{\prime \prime}=\left.\mathcal{A}\right|_{L}$.

If $d$ is odd, $L \in j_{\mathcal{D}}$ iff either $\left|\mathcal{A}^{\prime \prime}\right| \geq \frac{d+3}{2}$ or $\operatorname{det} \alpha_{L}=0$; where

$$
H^{0}\left(\mathcal{O}_{L}\left(\frac{d-1}{2}-\left|\mathcal{A}^{\prime \prime}\right|\right)\right) \stackrel{\alpha_{L}}{\rightarrow} H^{1}\left(\mathcal{O}_{L}\left(\left|\mathcal{A}^{\prime \prime}\right|-\frac{d+3}{2}\right)\right) .
$$

If $d$ is even, $L \in j_{\mathcal{D}}$ iff either $\left|\mathcal{A}^{\prime \prime}\right| \geq \frac{d+4}{2}$ or rank $\alpha_{L}<\frac{d}{2}-\left|\mathcal{A}^{\prime \prime}\right|$; where

$$
H^{0}\left(\mathcal{O}_{L}\left(\frac{d-2}{2}-\left|\mathcal{A}^{\prime \prime}\right|\right)\right) \stackrel{\alpha_{L}}{\rightarrow} H^{1}\left(\mathcal{O}_{L}\left(\left|\mathcal{A}^{\prime \prime}\right|-\frac{d+4}{2}\right)\right) .
$$

Proof. We prove the first statement. Since

$$
\left.i_{*} \mathcal{D}^{\prime \prime}\left(k_{\mathcal{D}}-1\right)\right|_{L} \simeq \mathcal{D}^{\prime \prime}\left(k_{\mathcal{D}}-1\right) \simeq \mathcal{O}_{L}\left(\frac{d-1}{2}-\left|\mathcal{A}^{\prime \prime}\right|\right)
$$

and $a_{1}+a_{2}=-2$, we have an exact sequence:

$$
0 \longrightarrow \mathcal{O}_{L}\left(\left|\mathcal{A}^{\prime \prime}\right|-\frac{d+3}{2}\right) \longrightarrow \mathcal{O}_{L}\left(a_{1}\right) \oplus \mathcal{O}_{L}\left(a_{2}\right) \longrightarrow \mathcal{O}_{L}\left(\frac{d-1}{2}-\left|\mathcal{A}^{\prime \prime}\right|\right) \longrightarrow 0
$$

From the long exact sequence in cohomology, it is obvious that $H^{0}\left(\left.\mathcal{D}_{\text {norm }}(-1)\right|_{L}\right) \neq$ 0 iff one of the two conditions of the theorem holds.

Example 4.8. Consider the following set of arrangements (where $\{z=0\}$ is the line at infinity): 


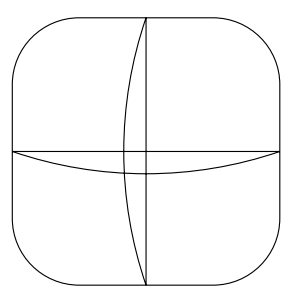

I

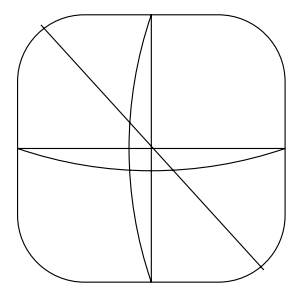

II

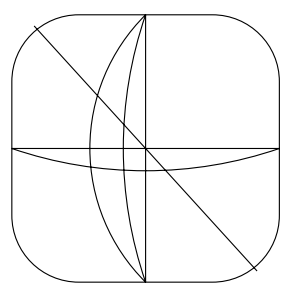

III

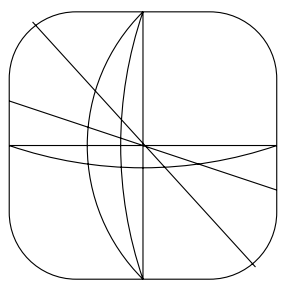

IV

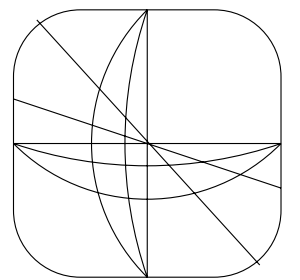

V

Arrangement I consists of the coordinate lines and the lines $\{y+z=0\},\{x+$ $z=0\} ; \mathcal{D} \simeq \mathcal{O}(-2)^{2}$ (which can be proved using Theorem 4.51 of [14]) so $\mathcal{D}$ is semistable. Arrangements II through V are obtained by adding (successively) the lines $\{x+y=0\},\{x+2 z=0\},\{x+2 y=0\},\{y+2 z=0\}$. Theorem 4.5 implies that the bundles associated to arrangements II, IV, and V are stable and the bundle associated to arrangement III is semistable. We can compute the Chern classes using Theorem 2.5:

$$
\begin{array}{ccc} 
& c_{1}\left(\mathcal{D}_{\text {norm }}\right) & c_{2}\left(\mathcal{D}_{\text {norm }}\right) \\
\text { I. } & 0 & 0 \\
\text { II. } & -1 & 1 \\
\text { III. } & 0 & 1 \\
\text { IV. } & -1 & 2 \\
\text { V. } & 0 & 3
\end{array}
$$

The normalized bundles fit into exact sequences:

$$
\begin{aligned}
& \text { I. } \quad 0 \quad \longrightarrow \quad \mathcal{O}^{2} \quad \longrightarrow \mathcal{D}_{\text {norm }} \longrightarrow 0 \\
& \text { II. } 0 \longrightarrow \mathcal{O}(-2) \longrightarrow \mathcal{O}(-1)^{3} \longrightarrow \mathcal{D}_{\text {norm }} \longrightarrow 0 \\
& \text { III. } 0 \longrightarrow \mathcal{O}(-2) \longrightarrow \mathcal{O}(-1)^{2} \oplus \mathcal{O} \longrightarrow \mathcal{D}_{\text {norm }} \longrightarrow 0 \\
& I V .0 \longrightarrow \mathcal{O}(-3) \longrightarrow \mathcal{O}(-1)^{2} \oplus \mathcal{O}(-2) \longrightarrow \mathcal{D}_{\text {norm }} \longrightarrow 0 \\
& V .0 \longrightarrow \mathcal{O}(-3) \longrightarrow \mathcal{O}(-1)^{3} \longrightarrow \mathcal{D}_{\text {norm }} \longrightarrow 0
\end{aligned}
$$

An easy application of the Beilinson spectral sequence [13] shows that a stable bundle with $c_{1}=-1$ and $c_{2}=1$ must be $\Omega_{\mathbb{P}^{2}}^{1}(1)$, so for arrangement II, the jump locus of $\mathcal{D}$ is empty. For arrangement III, Barth's theorem implies that the support of $j_{\mathcal{D}}$ is of degree one; by Theorem $4.7\{x+y=0\}$ and $\{y+z=0\}$ are jumping 
lines, hence $j_{\mathcal{D}}$ is the line $\{x-y+z=0\} \subseteq \mathbb{P}^{2^{\vee}}$. For arrangement IV, Theorem 4.7 shows that $\{y+z=0\}$ is a jumping line. In fact (Hulek, Prop. 8.2) a semistable two bundle $V$ with $c_{1}(V)=-1, c_{2}(V)=2$ has only a single jump line, so this is it!

Finally, for arrangement V, Barth's theorem implies that the jump locus is a cubic curve; Theorem 4.7 gives six lines of the arrangement which are jump lines. A computation shows that $\{x-y=0\},\{x-2 z=0\}$, and $\{y-z=0\}$ are also jump lines, so we have nine points in $\mathbb{P}^{2^{\vee}}$, which unfortunately only impose eight conditions on cubics. However, a final computation shows that the coordinate lines are not jump lines, which allows us to determine that the jump locus is a smooth cubic curve:

$$
4 x^{3}-2 x^{2} y-4 x y^{2}+2 y^{3}-4 x^{2} z-y^{2} z-x z^{2}-2 y z^{2}+z^{3}=0 .
$$

In [6], Dolgachev and Kapranov show that for generic arrangements in any dimension, a line contained in one of the hyperplanes of the arrangement is a jumping line; for line arrangements this also follows from Theorem 4.7. In fact, Dolgachev and Kapranov prove that for a generic arrangement with an odd number of lines, the points of the jump locus corresponding to the lines of the arrangement are singular points of fairly high multiplicity, which is an interesting contrast to the example above.

If $L \neq H$, then since $\mathcal{T}_{\text {or }}\left(i_{*} \mathcal{D}^{\prime \prime}, \mathcal{O}_{L}\right)=0$, we obtain an exact sequence:

$$
\left.\left.\left.0 \longrightarrow \mathcal{D}^{\prime}(-1)\right|_{L} \longrightarrow \mathcal{D}\right|_{L} \longrightarrow i_{*} \mathcal{D}^{\prime \prime}\right|_{L} \longrightarrow 0,
$$

with $\left.i_{*} \mathcal{D}^{\prime \prime}\right|_{L}$ torsion. If $d$ is odd, normalizing, restricting to $L$, and taking cohomology shows that $j_{\mathcal{D}^{\prime}} \subseteq j_{\mathcal{D}}$. In fact, we can do better. Let $L$ be a line which is not a line of the arrangement. Think of the arrangement as $\mathcal{A}^{\prime},\left|\mathcal{A}^{\prime}\right|=d$, and put $\mathcal{A}=\mathcal{A}^{\prime} \cup L$. Restricting to $L$ yields a long exact sequence

$$
\left.\left.\left.0 \longrightarrow \mathcal{T}_{\text {or }}\left(i_{*} \mathcal{D}^{\prime \prime}, \mathcal{O}_{L}\right) \longrightarrow \mathcal{D}^{\prime}(-1)\right|_{L} \longrightarrow \mathcal{D}\right|_{L} \longrightarrow i_{*} \mathcal{D}^{\prime \prime}\right|_{L} \longrightarrow 0
$$

In the previous theorem we studied a short exact sequence pruned from the right end of this type of complex; pruning a short exact sequence from the left end yields exact sequences:

$$
0 \longrightarrow \mathcal{O}_{L}\left(\frac{d-1}{2}-\left|\mathcal{A}^{\prime \prime}\right|\right) \longrightarrow \mathcal{O}_{L}\left(b_{1}\right) \oplus \mathcal{O}_{L}\left(b_{2}\right) \longrightarrow \mathcal{O}_{L}\left(\left|\mathcal{A}^{\prime \prime}\right|-\frac{d+3}{2}\right) \longrightarrow 0
$$

when $d$ is odd; in this case $b_{1}+b_{2}=-2$.

$$
0 \longrightarrow \mathcal{O}_{L}\left(\frac{d-2}{2}-\left|\mathcal{A}^{\prime \prime}\right|\right) \longrightarrow \mathcal{O}_{L}\left(a_{1}\right) \oplus \mathcal{O}_{L}\left(a_{2}\right) \longrightarrow \mathcal{O}_{L}\left(\left|\mathcal{A}^{\prime \prime}\right|-\frac{d+4}{2}\right) \longrightarrow 0
$$

when $d$ is even; in this case $a_{1}+a_{2}=-3$.

Theorem 4.9. Let $\mathcal{A}$ be an arrangement of $d$ lines, $L$ a line with $L \notin \mathcal{A}$ and $\mathcal{A}^{\prime \prime}=\left.\mathcal{A}\right|_{L}$. 
If $d$ is odd, then $L \in j_{\mathcal{D}}$ iff either $\frac{d-1}{2} \geq\left|\mathcal{A}^{\prime \prime}\right|$ or $\operatorname{det} \alpha_{L}=0$; where

$$
H^{0}\left(\mathcal{O}_{L}\left(\left|\mathcal{A}^{\prime \prime}\right|-\frac{d+3}{2}\right)\right) \stackrel{\alpha_{L}}{\rightarrow} H^{1}\left(\mathcal{O}_{L}\left(\frac{d-1}{2}-\left|\mathcal{A}^{\prime \prime}\right|\right)\right) .
$$

If $d$ is even, then $L \in j_{\mathcal{D}}$ iff either $\frac{d-2}{2} \geq\left|\mathcal{A}^{\prime \prime}\right|$ or rank $\alpha_{L} \leq\left(\left|\mathcal{A}^{\prime \prime}\right|-\frac{d+4}{2}\right)$; where

$$
H^{0}\left(\mathcal{O}_{L}\left(\left|\mathcal{A}^{\prime \prime}\right|-\frac{d+4}{2}\right)\right) \stackrel{\alpha_{L}}{\rightarrow} H^{1}\left(\mathcal{O}_{L}\left(\frac{d-2}{2}-\left|\mathcal{A}^{\prime \prime}\right|\right)\right) .
$$

Notice that the three jump lines of arrangement $V$ which are not lines of the arrangement are characterized by the first condition of the theorem. It seems reasonable to expect that the jump locus of the second kind is related to multiarrangements, about which very little is known (see Ziegler [25], or Solomon-Terao [19] for recent progress). We plan to return to this question in a later paper.

\section{Terao's conjecture}

One of the major open conjectures in the study of hyperplane arrangements is the following:

Conjecture 5.1. (Terao.) In characteristic zero, freeness of $D(\mathcal{A})$ depends only on the combinatorics of $\mathcal{A}$.

In [23], Yuzvinsky proves that for a fixed intersection lattice the set of free arrangements is Zariski open. In this section, we show that the vector bundle viewpoint has implications for Terao's conjecture. As noted earlier, $D(\mathcal{A})$ decomposes as $R(-1) \oplus D_{0}$, and the module $D_{0}$ has a minimal free resolution of the form (with $m \leq d-1$ ):

$$
0 \longrightarrow \bigoplus_{j=1}^{m-2} R\left(-\beta_{j}\right) \longrightarrow \bigoplus_{i=1}^{m} R\left(-\alpha_{i}\right) \longrightarrow D_{0} \longrightarrow 0 .
$$

If there is an $\alpha_{i}=1$, then the arrangement is a "near pencil", and we ignore this case, since such an arrangement is supersolvable [14]. The results of $\S 3$ imply

$$
\begin{aligned}
& 2 \leq \alpha_{i} \leq d-2 \\
& 3 \leq \beta_{j} \leq d-1 .
\end{aligned}
$$

Now fix an arrangement with intersection lattice $L_{\mathcal{A}}$ (which also fixes $\pi(\mathcal{A}, t)$ ). For Terao's conjecture we'll be interested in the situation where

$$
\pi(\mathcal{A}, t)=(1+t)(1+a t)(1+(d-1-a) t) .
$$

The results on the Jacobian ideal in $\S 2$ impose the additional conditions on the resolution:

$$
\sum \alpha_{i}-\sum \beta_{j}=d-1
$$




$$
\operatorname{deg} J_{Q}=\sum_{\substack{x \in L_{\mathcal{A}} \\ \operatorname{rank}(x)=2}} \mu(x)^{2}=(d-1)^{2}-a(d-1-a) .
$$

An easy computation shows the condition on $\operatorname{deg} J_{Q}$ is equivalent to

$$
\left(\begin{array}{c}
d-2 \\
2
\end{array}\right)-\sum_{i=1}^{m}\left(\begin{array}{c}
\alpha_{i}-1 \\
2
\end{array}\right)+\sum_{j=1}^{m-2}\left(\begin{array}{c}
\beta_{j}-1 \\
2
\end{array}\right)+1=a(d-1-a) .
$$

There are constraints on any free resolution of the form above; for example, if $\alpha_{1}=\min \left\{\alpha_{i}\right\}$ then

$$
\min \left\{\beta_{i}\right\} \geq \min \left\{\alpha_{2}, \ldots, \alpha_{m}\right\}+1
$$

This follows since any relation involves at least two generators, and must be a positive degree multiple of both. So if a counterexample to Terao's conjecture exists, it must be an integral solution of the above inequalities. Another constraint is the following, which follows easily by localization.

Lemma 5.2. There is a syzygy of degree $\geq \max \left\{\mu(x) \mid x \in L_{2}(\mathcal{A})\right\}$.

Example 5.3. Let $\mathcal{A}$ be an arrangement consisting of five lines through a point, and two additional lines in general position.

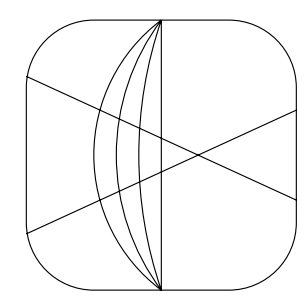

A quick check shows that $\pi(\mathcal{A}, t)=(1+t)(1+3 t)^{2}$. This is the simplest example of a nonfree arrangement where $\pi(\mathcal{A}, t)$ factors. Failure of freeness can be explained using the Addition-Deletion theorem of [14]; Lemma 5.2 provides another explanation. We combine the results above into

Theorem 5.4. Let $\mathcal{A}$ be an arrangement on d lines with intersection lattice $L_{\mathcal{A}}$, $M=\max \left\{\mu(x) \mid x \in L_{2}(\mathcal{A})\right\}$ and $\pi(\mathcal{A}, t)=(1+t)(1+a t)(1+(d-1-a) t)$. For

$$
\left\{\alpha_{1} \leq \alpha_{2} \leq \cdots \leq \alpha_{m}\right\} \in \mathbb{N}^{m} \text { and }\left\{\beta_{1} \leq \beta_{2} \leq \cdots \leq \beta_{m-2}\right\} \in \mathbb{N}^{m-2} \text {, }
$$

a unique integral solution to the following inequalities implies that Terao's conjecture holds for arrangements with intersection lattice $L_{\mathcal{A}}$.

1. The bound on the number of generators of $D_{0}$ :

$$
2 \leq m \leq d-1
$$


2. Global geometric constraints on the Jacobian ideal:

$$
\begin{gathered}
\sum_{i=1}^{m} \alpha_{i}-\sum_{j=1}^{m-2} \beta_{j}=d-1 \\
\left(\begin{array}{c}
d-2 \\
2
\end{array}\right)-\sum_{i=1}^{m}\left(\begin{array}{c}
\alpha_{i}-1 \\
2
\end{array}\right)+\sum_{j=1}^{m-2}\left(\begin{array}{c}
\beta_{j}-1 \\
2
\end{array}\right)+1=a(d-1-a) .
\end{gathered}
$$

3. Regularity constraints:

$$
\begin{aligned}
& 2 \leq \alpha_{i} \leq d-2 \\
& 3 \leq \beta_{j} \leq d-1
\end{aligned}
$$

4. Resolution constraints:

$$
\beta_{1} \geq \alpha_{2}+1
$$

5. Local geometric constraints:

$$
\exists \alpha_{i} \geq M
$$

Example 5.5. The non-Fano arrangement is an arrangement of seven lines with $\pi(\mathcal{A}, t)=(1+t)(1+3 t)^{2}$ :

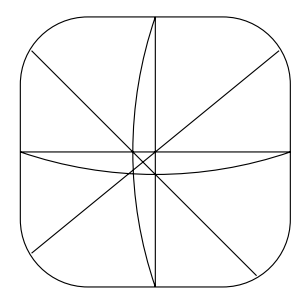

It is the smallest free arrangement which is not supersolvable. Applying Theorem 5.4, we find that there are nineteen different numerical possibilities for the free resolution of $D_{0}$. To shorten this list, we employ stability.

Write $\mathcal{A} \equiv \mathcal{B}$ if $\mathcal{A}$ and $\mathcal{B}$ have isomorphic intersection lattices, and let $\mathcal{D}_{\mathcal{A}}$, $\mathcal{D}_{\mathcal{B}}$ be the bundles associated to arrangements $\mathcal{A}$ and $\mathcal{B}$. Call a split two bundle $\mathcal{F}$ balanced if $\mathcal{F} \simeq \mathcal{O}_{\mathbb{p}^{2}}^{2}(a)$; a rank two bundle which splits is semistable iff it is balanced. It is obvious that the only free line arrangements which can be semistable are those with an odd number of lines, so for the remainder of this section we'll assume the number of lines is odd. Finally, note that the normalization of a balanced split two bundle is just $\mathcal{O}_{\mathbb{P}^{2}}$; so both Chern classes of the normalized bundle are zero.

Lemma 5.6. Suppose $\mathcal{D}_{\mathcal{A}}$ is balanced, and $\mathcal{B} \equiv \mathcal{A}$ is a counterexample to Terao's conjecture. Then $\mathcal{D}_{\mathcal{B}}$ is not semistable. In particular, there must be a syzygy on the Jacobian ideal of $\mathcal{B}$ of degree $<(d-1) / 2$. 
Proof. Once we've fixed a lattice, we have also fixed the Chern classes. Thus, a counterexample to Terao's conjecture, if semistable, would have a jump locus of degree $c_{2}=0$ by Barth's theorem, so a semistable counterexample would be a uniform bundle. But a uniform two bundle on $\mathbb{P}^{2}$ which does not split is $\mathcal{T}^{1}(a)$ ([21]), and these possibilities are excluded simply by considering the Chern classes. So we know that a counterexample must be unstable. Now from Lemma 4.4 we see that $H^{0}\left(\mathcal{D}_{\mathcal{B} \text { norm }}(-1)\right) \neq 0$, which implies the result about the syzygies.

Lemma 5.7. Any counterexample $\mathcal{B}\left(\equiv \mathcal{A}\right.$ with $\mathcal{D}_{\mathcal{A}}$ balanced $)$ to Terao's conjecture must have a syzygy on the Jacobian ideal of $\mathcal{B}$ of degree $>(d-1) / 2$.

Proof. If not, then $\mathcal{D}_{\mathcal{B} \text { norm }}$ would be generated by global sections. But a globally generated bundle with $c_{1}=0$ is trivial $([13]$ p. 53).

Combining these lemmas yields the following theorem. Notice that since having syzygies of low degree corresponds to being in special position, this is consistent with Yuzvinsky's results.

Theorem 5.8. Suppose a free arrangement $\mathcal{A}$ on $d$ lines has $\mathcal{D}_{\mathcal{A}} \simeq \mathcal{O}^{2}((d-1) / 2)$. A counterexample $\mathcal{B} \equiv \mathcal{A}$ to Terao's conjecture must have a syzygy of degree < $(d-1) / 2$, and also a syzygy of degree $>(d-1) / 2$.

Example 5.9. We return to case where $\mathcal{A}$ is the non-Fano arrangement. Of the nineteen numerically possible free resolutions for $D_{0}$, one corresponds to the case where $\mathcal{A}$ is free. Theorem 5.8 allows us to rule out fifteen of the other possibilities. Thus, there are only three numerical types of resolution possible if $\mathcal{A}$ is not free:

$$
\begin{gathered}
0 \longrightarrow R(-6) \longrightarrow R^{2}(-5) \oplus R(-2) \longrightarrow D_{0} \longrightarrow 0 \\
0 \longrightarrow R(-6) \oplus R^{3}(-3) \longrightarrow R(-5) \oplus R^{3}(-4) \oplus R^{2}(-2) \longrightarrow D_{0} \longrightarrow 0 \\
0 \longrightarrow R^{2}(-6) \oplus R^{2}(-3) \longrightarrow R^{4}(-5) \oplus R^{2}(-2) \longrightarrow D_{0} \longrightarrow 0
\end{gathered}
$$

If there are only two quadratic first syzygies, as in the last two cases, there can be at most one linear second syzygy, so these resolutions cannot occur. The first possibility actually does occur as the free resolution for an arrangement on seven lines with Poincaré polynomial $\pi(\mathcal{A}, t)=(1+t)(1+3 t)^{2}-$ it is the free resolution for the arrangement in Example 5.3. However, it cannot be the free resolution for the non-Fano arrangement. To see this, start out with arrangement $I$ from last section, and apply Theorem 3.4 twice. The regularity can be at most four, whereas this resolution has regularity five. Hence, any arrangement combinatorially equivalent to the non-Fano arrangement must be free. Remark: Theorem 4.51 of [14] and a similar sequence of subarrangements can also be used to obtain this result. 
Acknowledgement. I thank two anonymous referees for useful suggestions. The Macaulay2 software package was used to perform all computations.

\section{References}

[1] W. Barth, Some properties of stable rank two vector bundles on $\mathbb{P}^{n}$, Mathematische Annalen 226 (1977), 125-150.

[2] F. Bogomolov, Unstable vector bundles and curves on surfaces, Proceedings of the International Congress of Mathematicians (Helsinki, 1978), 517-524, Acad. Sci. Fennica, Helsinki, 1980 .

[3] A. Choudary and A. Dimca, Koszul complexes and hypersurface singularities, Proc. Amer. Math. Soc. 121 (1994), 1009-1016.

[4] H. Derksen and J. Sidman, Castelnuovo-Mumford regularity by approximation, math.AC/0211279.

[5] I. Dolgachev and M. Kapranov, Schur quadrics, cubic surfaces, and rank two vector bundles over the Projective Plane, Astérisque 218 (1993), 111-144.

[6] I. Dolgachev and M. Kapranov, Arrangements of Hyperplanes and Vector Bundles on $P^{n}$, Duke Mathematical Journal 71 (1993), 633-664.

[7] D. Eisenbud, Commutative Algebra with a view towards Algebraic Geometry, Graduate Texts in Mathematics, vol. 150, Springer-Verlag, Berlin-Heidelberg-New York, 1995.

[8] R. Friedman, Algebraic Surfaces and Holomorphic Vector Bundles, Springer-Verlag, BerlinHeidelberg-New York, 1998.

[9] R. Hartshorne, Algebraic Geometry, Graduate Texts in Mathematics, vol. 52, SpringerVerlag, Berlin-Heidelberg-New York, 1977.

[10] K. Hulek, Stable rank two vector bundles on $\mathbb{P}^{2}$ with $c_{1}$ odd, Mathematische Annalen 242 (1979), 241-266.

[11] G. Jiang and J. Feng, An algorithm to produce the structure sequence of an arrangement, in: Q. Lu, S. S. T. Yau and A. Libgober, eds., Singularities and complex geometry, 83-92, AMS/IP Studies in Advanced Mathematics, 5, 1997.

[12] M. Mustaţă and H. Schenck, The module of logarithmic $p$-forms of a locally free arrangement, J. Algebra 241 (2001), 699-719.

[13] C. Okonek, M. Schneider and H. Spindler, Vector Bundles on Complex Projective Spaces, Progress in Mathematics, vol. 3, Birkhäuser, Boston, 1980.

[14] P. Orlik and H. Terao, Arrangements of Hyperplanes, Grundlehren Math. Wiss., Bd. 300, Springer-Verlag, Berlin-Heidelberg-New York, 1992.

[15] L. Rose and H. Terao, A free resolution for the module of logarithmic forms of a generic arrangement, Journal of Algebra 136 (1991), 376-400.

[16] H. Schenck, A rank two vector bundle associated to a three arrangement, and its Chern polynomial, Advances in Mathematics 149 (2000), 214-229.

[17] R. Schwarzenberger, Vector bundles on the projective plane, Proceedings of the London Mathematical Society 11 (1961), 623-640.

[18] J. P. Serre, Sur les modules projectifs, Seminar Dubreil-Pisot, expose 2, 1960-61.

[19] L. Solomon and H. Terao, The double Coxeter arrangement, Commentarii Mathematici Helvetici 73 (1998), 237-258.

[20] H. Terao, Generalized exponents of a free arrangement of hyperplanes and Shepard-ToddBrieskorn formula, Inventiones Mathematicae 63 (1981), 159-179.

[21] A. Van de Ven, On uniform vector bundles, Mathematische Annalen 195 (1972), 245-248.

[22] S. Yuzvinsky, A free resolution for the module of derivations for generic arrangements, Journal of Algebra 136 (1991), 432-438.

[23] S. Yuzvinsky, Free and locally free arrangements with a given intersection lattice, Proceedings of the A.M.S. 118 (1993), 745-752. 
[24] S. Yuzvinsky, On generators of the module of logarithmic 1-forms with poles along an arrangement, Journal of Algebraic Combinatorics 4 (1993), 253-269.

[25] G. Ziegler, Multiarrangements of hyperplanes and their freeness, Contemporary Mathematics 90, 345-359. AMS, Providence, 1989

[26] G. Ziegler, Combinatorial construction of logarithmic differential forms, Advances in Mathematics 76 (1989), 116-154.

H. K. Schenck

Texas A\&M University

College Station, TX 77843

USA

e-mail: schenck@math.tamu.edu

(Received: August 3, 2001)

(D) To access this journal online:

(2P) http://www.birkhauser.ch 